OPEN ACCESS

Edited by:

Yi-Wei Tang,

Memorial Sloan Kettering Cancer

Center, United States

Reviewed by:

Hasan Ejaz,

Al Jouf University, Saudi Arabia

Zhiyong Zong,

Sichuan University, China

${ }^{*}$ Correspondence:

Po-Ren Hsueh

hsporen@ntu.edu.tw

Specialty section

This article was submitted to

Antimicrobials, Resistance

and Chemotherapy,

a section of the journal

Frontiers in Microbiology

Received: 05 October 2018 Accepted: 12 November 2018 Published: 27 November 2018

Citation:

Jean S-S, Lee N-Y, Tang H-J, Lu M-C, Ko W-C and Hsueh P-R

(2018) Carbapenem-Resistant

Enterobacteriaceae Infections: Taiwan

Aspects. Front. Microbiol. 9:2888.

doi: 10.3389/fmicb.2018.02888

\section{Carbapenem-Resistant Enterobacteriaceae Infections: Taiwan Aspects}

\author{
Shio-Shin Jean ${ }^{1,2}$, Nan-Yao Lee ${ }^{3}$, Hung-Jen Tang ${ }^{4,5}$, Min-Chi Lu ${ }^{6}$, Wen-Chien Ko ${ }^{3}$ and \\ Po-Ren Hsueh ${ }^{7,8 *}$
}

'Department of Emergency, School of Medicine, College of Medicine, Taipei Medical University, Taipei, Taiwan, ${ }^{2}$ Department of Emergency Medicine and Department of Emergency and Critical Care Medicine, Wan Fang Hospital, Taipei Medical University, Taipei, Taiwan, ${ }^{3}$ Department of Internal Medicine, National Cheng Kung University Medical College and Hospital, Tainan, Taiwan, ${ }^{4}$ Department of Medicine, Chi Mei Medical Center, Tainan, Taiwan, ${ }^{5}$ Department of Health and Nutrition, Chia Nan University of Pharmacy and Science, Tainan, Taiwan, ${ }^{6}$ Department of Microbiology and Immunology, School of Medicine, China Medical University, Taichung, Taiwan, ${ }^{7}$ Department of Laboratory Medicine, National Taiwan University Hospital, National Taiwan University College of Medicine, Taipei, Taiwan, ${ }^{8}$ Department Internal Medicine, National Taiwan University Hospital, National Taiwan University College of Medicine, Taipei, Taiwan

Carbapenem-resistant Enterobacteriaceae (CRE), a major resistance concern emerging during the last decade because of significantly compromising the efficacy of carbapenem agents, has currently become an important focus of infection control. Many investigations have shown a high association of CRE infections with high case-fatality rates. In Taiwan, a few surveys observed that a significant proportion (29-47\%) of the CR-Klebsiella pneumoniae isolates harbored a plasmidic allele encoding K. pneumoniae carbapenemases (KPC, especially KPC-2). A significant increase in the number of oxacillinase (OXA)-48-like carbapenemases among CR-K. pneumoniae isolates was observed between 2012 and 2015. By striking contrast, isolates of CREscherichia coli and CR-Enterobacter species in Taiwan had a much lower percentage of carbapenemase production than CR-K. pneumoniae isolates. This differs from isolates found in China as well as in the India subcontinent. Apart from the hospital setting, CRE was also cultured from the inpatients from communities or long-term care facilities (LTCF). Therefore, implementation of regular CRE screening of LTCF residents, strict disinfectant use in nursing homes and hospital settings, and appropriate control of antibiotic prescriptions is suggested to alleviate the spread of clinical CRE isolates in Taiwan. Although there are some promising new antibiotics against CRE, such as ceftazidime-avibactam, meropenem-vaborbactam, aztreonam-avibactam and cefiderocol, these agents are not available in Taiwan currently. Therefore, in order to effectively decrease case-fatality rates among patients with the infections owing to carbapenemase-producing CRE isolates, combination antibiotic schemes, including colistin (or amikacin) and/or tigecycline in combination with an anti-pseudomonal carbapenem agent, remain the mainstay for treating clinical CRE infections.

Keywords: carbapenem-resistant Enterobacteriaceae, carbapenemase, Klebsiella pneumoniae, Escherichia coli, long-term care facility, tigecycline, colistin, avibactam 


\section{INTRODUCTION}

Carbapenem-resistant Enterobacteriaceae (CRE), drawing great attention because of their serious resistance spectra and outbreak episodes in the northeastern United States since about two decades ago, have shown a high potential of rapid disseminations (Bratu et al., 2005a). Infections caused by CRE with production of various carbapenemases have become a major global worrisome concern due to their association with high $(\geq 30 \%)$ case-fatality rates in the current antibiotic pipeline (Tumbarello et al., 2012; Tzouvelekis et al., 2012; Navarro-San Francisco et al., 2013; Jean et al., 2015). In addition, McConville et al. conducted an observational study investigating CRE colonization (by rectal swabs) with subsequent impact on clinical outcomes for patients requiring admission to an intensive care unit (ICU) (McConville et al., 2017). By multivariate analysis, they found that CRE colonization also independently predicted development of a further systemic CRE infection at 30 days [adjusted odds ratio (aOR), 10.8; 95\% confidence interval (CI), 2.8-41.9, $P=0.0006]$. In 2017, the World Health Organization ranked CRE one of the first antibiotic-resistant "critical priority pathogens" (World Health Organization [WHO], 2017). Nevertheless, among these CRE isolates, the carbapenem non-susceptibility phenotypes are attributed to production of carbapenemase(s), or more likely, production of extended-spectrum $\beta$-lactamase (ESBL) plus AmpC $\beta$-lactamase with dysfunctional entry routes (i.e., porin change) of carbapenems, integrons and insertion sequence common region 1 (ISCR1) carrying various resistance genes, and/or efflux pumps, etc. (Doumith et al., 2009; Yang et al., 2012; Rui et al., 2018). To alleviate the resistance burden, close monitoring of CRE prevalence rates, as well as investigation of carbapenemase types, has become the main foci for infection control in most countries (Gupta et al., 2011).

Between 2004 and 2013, the prevalence rates of CRE among all clinical Enterobacteriaceae isolates have gradually risen (up to about $7 \%$ ) in medical centers and major regional teaching hospitals throughout Taiwan. However, official surveillance data, conducted by Taiwan Centers for Disease Control (Taiwan CDC), clearly showed the annual prevalence rates of overall CRE isolates collected from ICUs rose from $3.7 \%$ in 2008 to $15.3 \%$ in 2017 (data until the third quarter) (Taiwan Nosocomial Infection Surveillance [TNIS], 2017). In addition, among Escherichia coli isolates acquired at ICUs, CRE rates rose from $1.2 \%$ in 2008 to $4.0 \%$ in 2017 at medical centers (bed number, $>1000$ ) (until the third quarter), while CRE rates in major regional teaching hospitals (bed number, $500-1000$ ) rose from $1.0 \%$ in 2008 to $2.8 \%$ in 2017. These data might reflect the differences in CRE burden between hospitals of various care levels. By striking contrast, during the same period, significantly higher CRE rates for ICUacquired Klebsiella pneumoniae isolates were observed in medical centers (from 6.1 to $28.2 \%$ ) and in major regional teaching hospitals (from 3.7 to $24.8 \%$ ) than those of ICU-acquired E. coli (Taiwan Nosocomial Infection Surveillance [TNIS], 2017). It was determined by multivariate logistic regression analyses that clinicians in Taiwan (aOR, 1.73; 95\% CI, 1.03-2.92) and in the US (aOR, 1.89; 95\% CI, 1.05-3.39) are more likely to prescribe carbapenem antibiotics to treat the potential ESBL-producing
Enterobacteriaceae infections instead of $\beta$-lactam combination agents compared to other countries (Harris et al., 2017). Clinical overuse of carbapenem agents unavoidably result in increased drug resistance (Tumbarello et al., 2012; Sheu et al., 2018).

\section{STUDY DESIGNS}

We searched and reviewed literature from the 2002-2018 PubMed database containing important keywords, including "carbapenem-resistant Enterobacteriaceae," "carbapenemaseproducing Enterobacteriaceae," "prevalence rates," "mortality," "case-fatality," “Taiwan," "Centers for Disease Control," "Klebsiella pneumoniae," "Klebsiella pneumoniae carbapenemase (KPC)," "New Delhi metallo- $\beta$-lactamase (NDM)," "oxacillinase48 (OXA-48)-like," "Escherichia coli," "Enterobacter species," "resistance mechanisms," "community-acquired," "long-term care facility," "Clinical and Laboratory Standards Institute," "European Committee on Antimicrobial Susceptibility Testing," "monotherapy," "combination therapy," "tigecycline," "colistin," and "novel antibiotics."

\section{PREVALENCE AND MORTALITY RATES OF CARBAPENEMASE-PRODUCING ENTEROBACTERIACEAE (CPE) AMONG CRE}

In Pakistan, spread of special sequence types (STs; ST15 and ST48) of multidrug-resistant (MDR) Klebsiella species (most were ESBL-producing K. quasipneumoniae) isolates was observed since 2010 at one tertiary-care hospital (Ejaz et al., 2017). Similarly, among 83 carbapenem-resistant K. pneumoniae isolates studied in Brazil, a clinical isolate of KPC-2 and OKP-B-6 $\beta$-lactamase-producing $K$. quasipneumoniae subsp. similipneumoniae was also reported. (Nicolás et al., 2018). By stark contrast, global dissemination of CPE (especially $K$. pneumoniae isolates) in fact has been occurring at an alarming pace for many years (Logan and Weinstein, 2017). In Taiwan, most CP-K. pneumoniae isolates are the KPC producers (Jean et al., 2015). The bla $a_{\mathrm{KPC}}$ genes mostly reside on transferable plasmids which contain transposase, resolvase, and mobile transposons (Tn4401), thereby posing a formidable threat to infection control (Nordmann et al., 2009). As compared to other Enterobacteriaceae species, it is noteworthy that these mobile transposons are only detected among few epidemic clones of KPC producers of $K$. pneumoniae with distinct STs (predominantly ST258 in US and Israel, while ST11 in China and Taiwan) (Naas et al., 2008). The ST11 clone was the most prevalent ESBLproducing clone (particularly CTX-M-15, CTX-M-14, and SHV5 types) of K. pneumoniae isolates in many Asian countries (Lee et al., 2011; Ma et al., 2013a).

Regarding the molecular analyses of carbapenemase types in CRE in Taiwan, a multicenter study first examined imipenemresistant K. pneumoniae isolates collected from 13 Taiwanese hospitals from 2002 through 2009 (Ma et al., 2013a). This study revealed that a majority (84.6\%) of K. pneumoniae strains (mainly 
ST11 clone) with $>2 \mathrm{mg} / \mathrm{L}$ minimum inhibitory concentrations (MICs) to imipenem harbored $b$ la $_{\mathrm{IMP}-8}$, plus various genes encoding ESBLs in combination with loss of porins (mainly OmpK35) (Ma et al., 2013a). In 2012, a small-scale Taiwanese study, conducted by Chang et al., investigated 66 patients with CRE (comprising K. pneumoniae and E. coli) infections $(n=46)$ or colonization $(n=20)$. It showed that the CPE prevalence rate was $28.8 \%\left(n=19 ; 14\right.$ of which harbored $\left.b l a_{\mathrm{KPC}-2}\right)$, and a 30 -day mortality rate was $50 \%(23 / 46)$ among patients with CRE infections (Chang et al., 2015). The CPE prevalence of Chang's study was different from that [11.5\%; most were metallo$\beta$-lactamase $(\mathrm{M} \beta \mathrm{L})$ producers] of the study conducted by Javed et al. (2016) in Pakistan during 2013-2014. In addition, this mortality rate was higher compared to that (40.8\%) of the other CRE study (susceptibility evaluated by the disk diffusion method, and data interpretation in accordance with the 2009 criteria) at a medical center located in the middle-western part of Taiwan during 2010-2011 (Huang et al., 2014). In Chang's study, nearly one-half $[47.8 \%(22 / 46)]$ of the infections originated in the lower respiratory tract. Furthermore, patients with a co-morbidity of diabetes mellitus, initially presenting with shock or high scores of the Acute Physiology and Chronic Health Evaluation (>23 points), or receiving non-susceptible antibiotic regimen therapy (regardless of single or combination drug therapy) for $>48 \mathrm{~h}$

TABLE 1 | Studies of carbapenem-resistant Enterobacteriaceae in Taiwan and other countries.

\begin{tabular}{|c|c|c|c|c|}
\hline Study periods & $\begin{array}{l}\text { CRE case } \\
\text { numbers, or CRE } \\
\text { isolates under } \\
\text { survey }\end{array}$ & $\begin{array}{c}\text { Carbapenemase } \\
\text { production, \% }\end{array}$ & Outcomes & Reference \\
\hline 2010-2012 & $\begin{array}{l}\text { 1,135 carbapenem } \\
\text { non-susceptible } \\
\text { Enterobacteriaceae } \\
\text { isolates (from } \\
\text { various clinical } \\
\text { sources) }\end{array}$ & $5 \%(57 / 1135)$ & NA & Wang et al., 2015 \\
\hline 2012 & $\begin{array}{l}66 \text { (including } 46 \\
\text { patients with } \\
\text { diverse CRE } \\
\text { infections and } 20 \\
\text { patients with CRE } \\
\text { colonization) }\end{array}$ & $28.8 \%(19 / 66)$ & $\begin{array}{l}\text { In-hospital } \\
\text { mortality, } 50 \% \\
\text { (23/46) for patients } \\
\text { with CRE infections }\end{array}$ & Chang et al., 2015 \\
\hline 2012-2013 & $\begin{array}{l}105 \text { (including } 49 \\
\text { patients with } \\
\text { various CRE } \\
\text { infections, and } 56 \\
\text { patients with CRE } \\
\text { colonization) }\end{array}$ & $29.5 \%(31 / 105)$ & $\begin{array}{l}\text { Overall in-hospital } \\
\text { mortality, } 43.8 \% \\
\text { (46/105); for } 49 \\
\text { cases with CRE } \\
\text { infections, } 63.3 \% \\
(31 / 49)\end{array}$ & Wu et al., 2016 \\
\hline 2012-2015 & $\begin{array}{l}1,457 \\
\text { CR-K. pneumoniae } \\
\text { isolates }\end{array}$ & $31.4 \%(457 / 1457)$ & NA & Chiu et al., 2018 \\
\hline 2017 & $\begin{array}{l}85 \text { bloodstream } \\
\text { CRE isolates, } \\
\text { comprising } \\
\text { Escherichia coli } \\
(n=14) \text { as well as } \\
\text { K. pneumoniae } \\
(n=71)\end{array}$ & $\begin{array}{c}41.2 \%(35 / 85) ; \\
46.5 \%(33 / 71) \text { for } \\
\text { CR-K. pneumoniae } \\
\text { isolates }\end{array}$ & NA & Jean et al., 2018b \\
\hline 2015 & $\begin{array}{l}70 \text { CRE isolates } \\
\text { (from various } \\
\text { clinical sources), } \\
\text { collected from } \\
\text { patients in Brazil }\end{array}$ & $\begin{array}{l}80 \%(56 / 70) ; \text { all } \\
\text { harbored bla } \mathrm{KPC}\end{array}$ & NA & $\begin{array}{l}\text { Lorenzoni et al., } \\
2017\end{array}$ \\
\hline 2016-2017 & $\begin{array}{l}22 \text { CRE isolates, } \\
\text { collected from urine } \\
\text { samples of patients } \\
\text { in the United } \\
\text { Kingdom }\end{array}$ & $45.4 \%(10 / 22)$ & NA & $\begin{array}{l}\text { Woodford et al., } \\
2017\end{array}$ \\
\hline 2017 & $\begin{array}{l}287 \text { clinical CRE } \\
\text { isolates, collected } \\
\text { from patients in } \\
\text { Thailand }\end{array}$ & $\begin{array}{c}77.7 \%(223 / 287), \text { a } \\
\text { majority of them } \\
\text { harbored bla }{ }_{N D M-1}\end{array}$ & NA & Laolerd et al., 2018 \\
\hline
\end{tabular}

CRE, carbapenem-resistant Enterobacteriaceae. NA, non-applicable. 


\section{-CPE/CRE, $\% \quad$ KPCs/CPE, $\% \quad$ KPC-2/CPE, \% $\square$ OXA-48-like/CPE, $\%$}

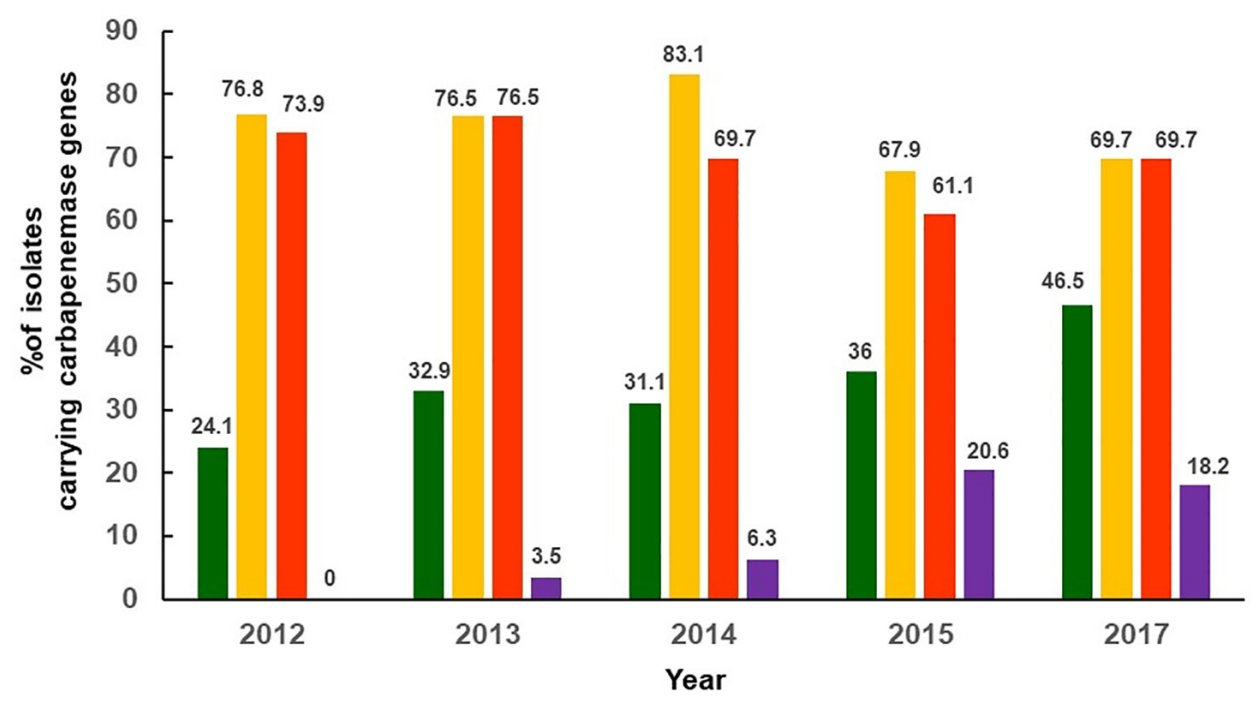

FIGURE 1 | Distribution of carbapenemases. Annual proportions of overall and different types of carbapenemase producers (Klebsiella pneumoniae carbapenemase, oxacillinsase-48-like) among carbapenem-resistant K. pneumoniae isolates collected between 2012 and 2017 in Taiwan (Chiu et al., 2018 ; Jean et al., 2018b).

had significantly higher case-fatality rates $(P<0.05)$ than the other factors by the univariate analysis (Chang et al., 2015). Of note, patients who acquired CRE infections owing to KPC (dominant carbapenemase type)-producing Enterobacteriaceae also had a trend toward more fatal outcomes than those without KPC ( $P=0.14)$ (Chang et al., 2015). An additional CRE study conducted at a single medical center of northern Taiwan during 2012-2013 showed that a CRE isolate with an imipenem $\mathrm{MIC} \geq 16 \mathrm{mg} / \mathrm{L}$ independently predicted 14-day mortality among patients regardless if the isolate was from infection or colonization ( $\mathrm{Wu}$ et al., 2016). In the survey published by $\mathrm{Wu}$ et al., KPC-2 was the dominant (87.1\%) carbapenemase among CPE (29.5\% of overall CRE), and the in-hospital mortality rate among patients with CR-K. pneumoniae was $43.8 \%$ (Wu et al., 2016). Wang et al. examined 1135 clinical isolates of various Enterobacteriaceae species collected from four major hospitals in Taiwan between 2010 and 2012. Fifty-seven isolates were carbapenemase-producing Enterobacteriaceae (CPE) isolates (5\%), 54.4\% of the CPE isolates co-harbored bla $a_{\mathrm{ESBL}}$ alleles. Furthermore, Wang et al. found that Enterobacter cloacae isolates predominantly harbored the $b l a_{\mathrm{IMP}-8}$ allele (26/27), while isolates of CP-K. pneumoniae mainly harbored bla $a_{\mathrm{KPC}-2}$ alleles (16/17; ST11 clone predominated) (Wang et al., 2015). In addition, among Taiwanese CRE isolates surveyed, approximately one-half (45.8\%) harbored various bla $a_{\mathrm{ESBL}}$ genes (80\% were the $b l a_{\mathrm{CTX}-\mathrm{M}}$ types), while $62.0 \%$ harbored alleles encoding various plasmid-mediated AmpC $\beta$-lactamases (70.6\% were $b l a_{\mathrm{DHA}}$ and $22.3 \%$ were $b l a_{\mathrm{CMY}}$ ) (Wang et al., 2015). The high rates of $K$. pneumoniae isolates co-harboring ESBL and/or AmpC-encoding alleles among CRE corresponded well with those of an Asia-Pacific study with respect to Enterobacteriaceae isolates responsible for complicated intra-abdominal infections
(cIAI) and complicated urinary tract infections (cUTI) from 2008 through 2014 (Jean et al., 2017). According to the above findings, instead of carbapenemase production, ESBL with concomitant AmpC production and membrane impermeability caused by porin loss obviously more likely confers in vitro non-susceptibility to carbapenem agents (especially ertapenem) among CRE in Taiwan and the Asia-Pacific region. These results are similar to that found in Hong Kong (Ho et al., 2016).

Despite non-CPE isolates account for a majority of CRE in Taiwan, an up-surging CPE trend [three-fourths (74.5\%) of CPE harboring $b l a_{\mathrm{KPC}-2}$ and were ST11 clone, followed by CPE harboring bla $a_{\mathrm{VIM}-1}$ allele $(12.7 \%)$, etc.] was observed by Chiu et al. (2013). The CPE rates have increased from $0 \%$ in 2010 to $22.3 \%$ in 2012 among Taiwanese CR-K. pneumoniae isolates (showing $>1 \mathrm{mg} / \mathrm{L}$ of MICs to imipenem or meropenem) (Chiu et al., 2013). Furthermore, it is noteworthy that the prevalence rates of $\mathrm{CPE}$ [36.4\%; mostly related to various bla $a_{\mathrm{KPC}}(75.9 \%)$, followed by bla $a_{\mathrm{OXA}-48}$-like $(8.8 \%)$, bla $a_{\mathrm{VIM}-1}$ $(7.9 \%)$, and bla $a_{\mathrm{IMP}-8}(5.7 \%)$, etc] among the studied CRK. pneumoniae isolates (approximately $99 \%$ were non-susceptible to imipenem, and $85.8 \%$ were collected from medical centers) were estimated to have 1.5-fold increase from 2012 through 2015. Most significantly, an increasing trend was observed in the middle-western part of Taiwan (Chiu et al., 2018). Data regarding important CRE studies in Taiwan and other countries are shown in Table 1. There were big variations in the rates of $\mathrm{CPE}$ among clinical CRE isolates in Taiwan as compared to those from other countries (Lorenzoni et al., 2017; Woodford et al., 2017; Laolerd et al., 2018). It is notable that persistently high KPC-2 rates (73.9\% in 2012, and 61.1\% in 2015) and emergence of KPC-17 as well as KPC-34 since 2014 were observed (Chiu et al., 2018). As the K. pneumoniae of ST11 clone accounted for 
86.9\% of overall CP-K. pneumoniae isolates (Chiu et al., 2018), heightened infection control maneuvers are absolutely warranted for Taiwanese medical centers. Figure 1 illustrates the annual percentages of overall carbapenemase producers, overall KPCs and KPC-2 producers, as well as OXA-48-like producers among clinical CR-K. pneumoniae isolates (mainly non-susceptible to imipenem and ertapenem) collected between 2012 and 2015 in Taiwan (Chiu et al., 2018; Jean et al., 2018b).

Carbapenem-resistant Enterobacteriaceae that only harbor

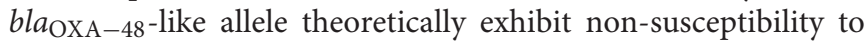
most carbapenem agents while sparing most $\beta$-lactam agents. However, most of the bla $a_{\mathrm{OXA}-48}$-like-harboring CRE (especially K. pneumoniae) isolates are actually also the ESBL and/or AmpC co-producers (Saïdani et al., 2012; Girlich et al., 2014). Consequently, many OXA-48-like-producing CRE strains show in vitro extensively drug-resistant phenotypes. In 2014, an OXA-48-like carbapenemase-producing E. coli strain, which concomitantly harbored bla $a_{\mathrm{CTX}-\mathrm{M}-1}$, was cultured from pus sample of a Cambodian patient who resided in Taiwan (Jao et al., 2017). An additional 2010 study from India regarding $\mathrm{CRE}$, some of which were $\mathrm{CPE}$ harboring $b l a_{\mathrm{NDM}}$, bla $a_{\mathrm{VIM}}$ or bla $a_{\text {OXA-181-like alleles, revealed that the case-fatality rate was }}$ $56.7 \%$ (Mariappan et al., 2017). A sharp rise in the annual prevalence rate of OXA-48-like production among Taiwanese CPE isolates between 2012 and 2015 was also observed (Chiu et al., 2018) (Figure 1). Spread of bla $a_{\mathrm{NDM}}$ alleles occurred rapidly in the Indian subcontinent during the last decade (Kumarasamy et al., 2010), and also in southeastern Asia (Vietnam and the Philippines) (Jean et al., 2017). Nevertheless, reports regarding the NDM-producing Enterobacteriaceae colonization in one patient ( $3 \mathrm{~K}$. pneumoniae isolates harboring $b l a_{\mathrm{NDM}-1}$, probably acquired from India) (Wu et al., 2010) and infection in the other patient (1 Klebsiella oxytoca isolate harboring bla $a_{\mathrm{NDM}-1}$, acquired from China) (Lai et al., 2011) in Taiwan were rare (Jean et al., 2017).

\section{COMMUNITY AND LONG-TERM CARE FACILITY (LTCF)-ACQUIRED CRE}

Few studies have addressed the percentage of community acquisition of clinical CRE infections in the PubMed database. However, a few investigations in Taiwan have addressed this important topic. A clinical study, conducted at a medical center located in southern Taiwan in 2015, showed approximately $30 \%$ $(23 / 78)$ of cases, with predominance of elderly female patients and infections originating in the urologic system, acquired CRE in a community setting (Tang et al., 2016). The percentage of community-acquired CRE was higher than that [12\% (14/117)] of another Taiwanese study conducted at a large medical center in $2010(P=0.002)$ (Lai et al., 2013), and also higher than that [5.6\% (17/305)] of an US-CRE study (Thaden et al., 2014). In the latter study, 91\% of overall CRE isolates were K. pneumoniae, while 59\% were cultured from urine between 2008 and 2012 $(P<0.0001)$ (Thaden et al., 2014). In addition, it was observed that many residents from the LTCF in Taiwan were also the carriers of CRE, most (24/27) were K. pneumoniae (Chuang,
2015; Mao et al., 2018). This was similar to the study conducted by Lee C.M. et al. (2017) who observed that a significant proportion of $K$. pneumoniae strains cultured from rectal swabs of LTCF residents were CRE (38.9 and 88.9\% showed in vitro non-susceptibility to ertapenem and imipenem, respectively). Therefore, in Taiwan, regular screening for CRE carriage (urine, sputum, and rectal swabs) among the LTCF residents to implement subsequent contact isolation precaution, hand hygiene, strict implementation of environmental disinfection (including equipment of LTCF), and adequate education to caregivers and family about the importance of infection control are beneficial to decrease CRE carriage rates of LTCF residents. All of these measures are crucial for preventing CRE spread at LTCF (Lee C.M. et al. (2017); Lai et al., 2018; Liu et al., 2018).

\section{SURVEILLANCE DATA OF ANTIMICROBIAL RESISTANCE IN TAIWAN IN 2017}

To fully explore the evolutionary trends of the annual resistance burden, the Taiwan CDC conducted a consecutive 4-year survey of antimicrobial resistance (AMR) regarding important clinical bacterial pathogens since 2017 (Jean et al., 2018b). Among 673 bloodstream K. pneumoniae isolates collected from 16 major teaching hospitals throughout Taiwan in 2017, 71 (10.5\%) displayed non-susceptibility to at least one carbapenem agent. In addition, a total of 92 (13.8\%) K. pneumoniae isolates exhibited ESBL phenotypes that were unrelated to KPC and/or NDM production [with a 92\% non-susceptible (NS) rate to piperacillin-tazobactam, $100 \%$ to ceftazidime, and $74 \%$ to cefepime]. Phenotypic ESBL isolates had high NS rates to ertapenem (40.2\%), tigecycline $[7.6 \%$ based on the US Food and Drug Administration (FDA) criteria, and 16.3\% based on the criteria recommended by the European Committee on Antimicrobial Susceptibility Testing (EUCAST) in 2018 (European Committee on Antimicrobial Susceptibility Testing [EUCAST], 2018)], amikacin (13.0\%), as well as ceftolozanetazobactam $[70.7 \%$ based on criteria recommended by Clinical, and Laboratory Standards Institute [CLSI], 2018, and 80.4\% based on the EUCAST 2018 criteria]. Of note, among K. pneumoniae blood isolates validated as CPE by multiplex PCR tests, 24 (3.6\%) harbored bla $a_{\mathrm{KPC}}$ allele (1 isolate showed susceptibility to all tested carbapenem agents), eight (33.3\%) of the KPC-producing $K$. pneumoniae isolates were acquired from a community setting. In addition, 7 (1.0\%) K. pneumoniae isolates harbored bla $a_{\mathrm{OXA}-48}$-like alleles ( 1 isolate showed susceptibility to all tested carbapenem agents, while 6 exhibited ESBL phenotypes), $1(0.15 \%)$ and $4(0.6 \%)$ isolates harbored $b l a_{\mathrm{NDM}}$ and $m c r-1$ gene, respectively. The rate of KPC-producing isolates [32.4\% (23/71)] among Taiwanese CR-K. pneumoniae isolates in 2017 was significantly higher than that $(2.8 \%)$ in the 2010-2012 Taiwanese study (Wang et al., 2015). Furthermore, out of the total KPC-producing $K$. pneumoniae isolates $(n=24)$, there were two $(8.3 \%)$ showing non-susceptibility to ceftazidimeavibactam, 5 (20.8\%) showing non-susceptibility to colistin, and $4(16.7 \%)$ displaying non-susceptibility to tigecycline based on 
the EUCAST 2018 criteria (Jean et al., 2018b, in Infect Drug Resist).

\section{CARBAPENEM RESISTANCE MECHANISMS AMONG CR-E. coli ISOLATES}

As compared to a much higher CPE rate among clinical isolates of $K$. pneumoniae, two Taiwanese studies concluded that coexistence of a plasmidic AmpC $\beta$-lactamase (DHA-1, CMY-2) in combination with loss of an outer membrane porin (OmpC/F) is the main mechanism responsible for non-susceptibility to carbapenems for CR-E. coli (Chia et al., 2009; Ma et al., 2013b). This finding differs from that in Mainland China [89\% of CRE. coli isolates $(n=164)$ harbored $b l a_{\mathrm{NDM}}(n=81)$ or bla $a_{\mathrm{KPC}}$ $(n=65)$ alleles] (Zhang et al., 2017), and in India (most CRE. coli harbored bla $a_{\mathrm{NDM}}$ with or without bla $a_{\mathrm{OXA}-48}$-like alleles) from 2011 through 2013 (Mohanty et al., 2017). Surveillance data from the Taiwan CDC-AMR 2017 showed a total of 686 E. coli blood isolates were collected. Among them, only 14 (2.0\%) isolates showed in vitro non-susceptibility to ertapenem. Of note,

TABLE 2 | Clinical efficacy of different antibiotic regimens on patients with clinical infections due to isolates of carbapenemase-producing Enterobacteriaceae.

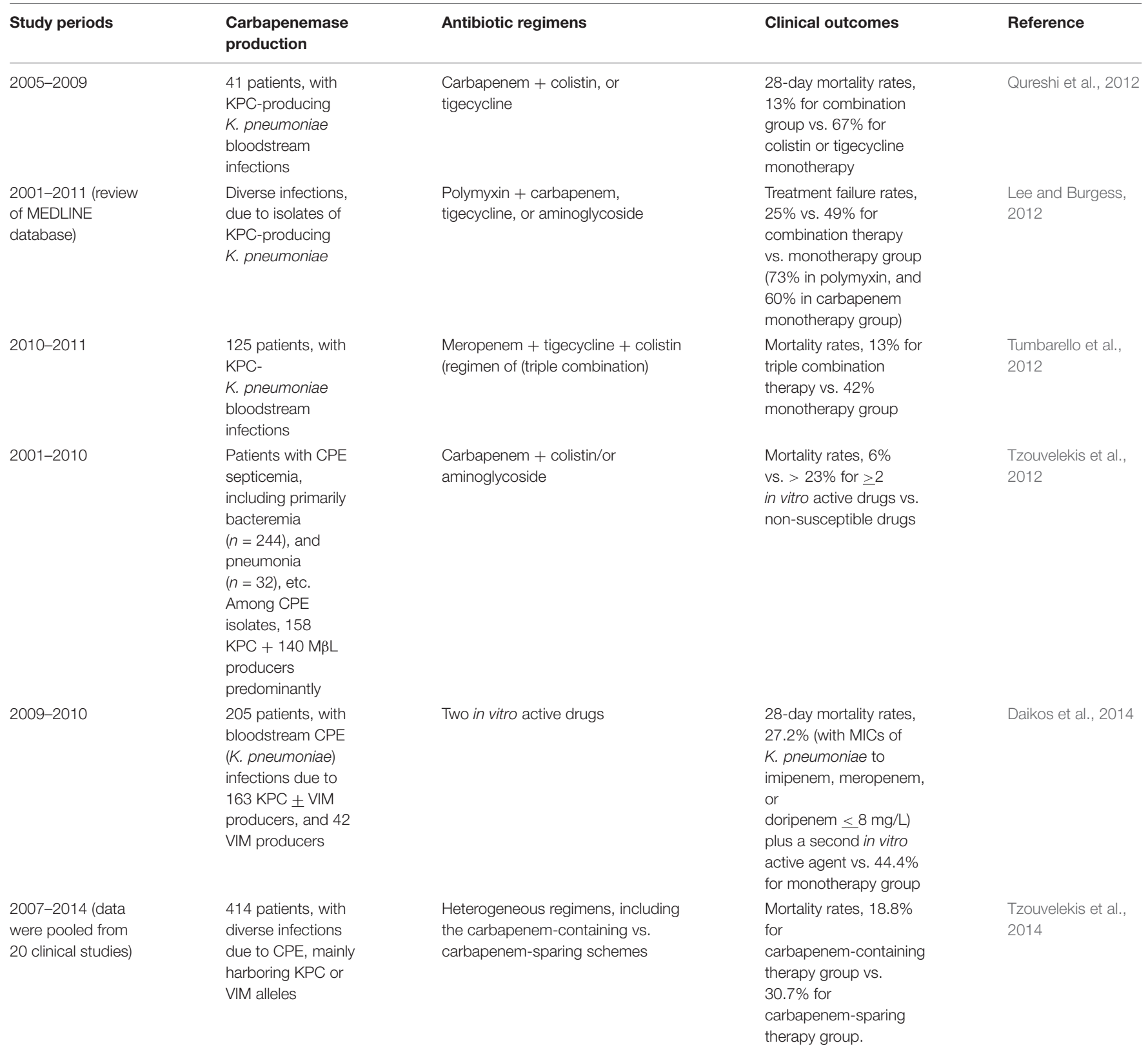

KPC, Klebsiella pneumoniae carbapenemase. CPE, carbapenemase-producing Enterobacteriaceae. MBL, metallo- $\beta$-lactamase. VIM, Verona integron-encoded metallo$\beta$-lactamases. 
$5(0.7 \%)$ isolates harbored the $m c r-1$ gene, similar to the one found in Mainland China (Quan et al., 2017), while the other two harbored a $b l a_{\mathrm{KPC}}$ and $b l a_{\mathrm{NDM}-1}$ allele, respectively.

\section{CARBAPENEM RESISTANCE MECHANISMS AMONG CR-Enterobacter spp. ISOLATES}

Fewer studies have examined mechanisms of non-susceptibility to carbapenem agents among isolates of Enterobacter species than those for $K$. pneumoniae in the PubMed database. A study focusing on ertapenem-resistant Enterobacter cloacae isolates (MIC > $2 \mathrm{mg} / \mathrm{L}$, in accordance with the CLSI 2009 criteria) collected in 2007 was conducted by Yang et al. (2012) in Taiwan. Analysis of porin expression, detection of efflux pumps and $\beta$-lactamase(s), as well as susceptibility tests were performed. Only a few isolates [3/53 (5.7\%)] harbored bla $a_{\mathrm{IMP}-8}$, while porin change (30-40\%) and efflux pump(s) $(\geq 70 \%)$ in combination with ESBL or AmpC significantly contributed to ertapenem non-susceptibility for Taiwanese ertapenem-resistant E. cloacae isolates. Because of geographic variations, production of different types of carbapenemases among the carbapenem (imipenem, ertapenem)-NS E. cloacae isolates was observed in India (VIM-2, VIM-6, and NDM-1) (Khajuria et al., 2014) and in Israel (KPC-2) (Marchaim et al., 2008).

\section{MONOTHERAPY VS. COMBINATION THERAPY FOR CRE INFECTION}

Patients with CPE infections are undoubtedly at an extremely high risk for inappropriate antibiotic therapy (Tumbarello et al., 2012; Chang et al., 2015). As stated earlier, tigecycline and colistin showed good in vitro susceptibility results against most $\mathrm{CRE}$ isolates and $\mathrm{KPC}$ or $\mathrm{M} \beta \mathrm{L}$-producing $\mathrm{CPE}$ isolates (Bratu et al., 2005b; Castanheira et al., 2008). As stated in the Taiwan CDC-AMR 2017 data, the tigecycline/colistin MIC values of one E. coli and one K. pneumoniae isolate harboring the $b l a_{\mathrm{NDM}}$ allele were $0.06 / 0.25 \mathrm{mg} / \mathrm{L}$ and $0.25 / 0.25 \mathrm{mg} / \mathrm{L}$, respectively. Nevertheless, to maximize the clinical effectiveness of colistin against impending resistant Gram-negative bacteria (GNB; i.e., MIC of colistin is $2 \mathrm{mg} / \mathrm{L}$ ), a study showed that both the loading, as well as maintenance dose should be increased (Garonzik et al., 2011). In addition, tigecycline was validated as a bacteriostatic agent with suboptimal concentrations in bloodstream $(<2 \mathrm{mg} / \mathrm{L})$ after administration of the standarddose regimen (Yahav et al., 2011). Consequently, monotherapy with tigecycline or colistin is not recommended in the treatment of severe GNB infections owing to high clinical failure and superinfection rates. Bass et al. found that combination therapy of multiple agents that have appropriate in vitro activities for $\geq 48 \mathrm{~h}$ was associated with improved survival rates (OR, 0.19 ; $95 \%$ CI, 0.06 to $0.56 ; P<0.01$ ) for critically ill patients with CR-GNB bacteremia (regardless of Enterobacteriaceae species or non-fermentative GNB) (Bass et al., 2015; Lee
C.H et al., 2017). Many combination therapies were also been shown to result in favorable outcomes in patients with CP-GNB. For example, a combination of doripenem with colistin was reported to have an excellent in vitro synergistic effect against CP-K. pneumoniae isolates (Jernigan et al., 2012; Rodríguez-Baño et al., 2018). In addition, a tigecyclinecolistin combination was also shown to be superior to colistin monotherapy in decreasing future resistance to colistin (Lee et al., 2009).

In Taiwan, data comparing therapeutic efficacy between different antibiotic regimens against CRE or CPE were scarce (Lee et al., 2011; Ku et al., 2017). Although the rate of tigecycline non-susceptibility among Taiwanese CRE (E. coli plus K. pneumoniae) blood isolates in 2017 was not high [3.5\% $(3 / 85)$ based on FDA criteria and $12.9 \%$ (11/85) based on the EUCAST 2018 criteria], a Taiwanese study aiming specifically at 16 tigecycline-resistant $K$. pneumoniae strains elucidated that $\mathrm{ramR}$ deficiency and/or widespread mutated tet(A) are the main mechanisms conferring non-susceptibility to the tetracyclineclass agents for these $K$. pneumoniae isolates (Chiu et al., 2017). In addition, acquisition of fosfomycin-resistant genes, fos $A$ subtypes and fos $C 2$, which were mainly transmitted by plasmids and/or transposons, was found in many of the Enterobacteriaceae species in Eastern Asian countries, including Taiwan (Yang et al., 2017). In addition, although amikacin, gentamicin and fosfomycin exhibited acceptable in vitro susceptibility rates against some CPE species (KPC and VIM) (Endimiani et al., 2010; Lorenzoni et al., 2017), these agents are recommended as mere adjunctive options against critical CP-GNB infections because of rapid induction of resistance after monotherapy (Tseng et al., 2017; Yang et al., 2017). By contrast, against the non-CP-CR-K. pneumoniae strains (with changes of outmembrane porins) collected from a medical center of southern Taiwan during 2008-2012, Ku et al. used the sub-inhibitory concentrations $(1 / 2 \times \mathrm{MIC})$ of antibiotics under evaluation and time-kill studies to analyze in vitro synergism between different combination regimens. As compared to tigecycline plus fosfomycin and colistin plus fosfomycin, $\mathrm{Ku}$ et al. observed that tigecycline in combination with colistin showed much better in vitro synergism, as well as bactericidal efficacy ( $\mathrm{Ku}$ et al., 2017).

\section{CLINICAL EVIDENCE OF COMBINED ANTIMICROBIAL SCHEMES AGAINST CPE INFECTIONS}

Although the above in vitro synergy data supported the use of combination regimens, well-designed clinical randomized control trials that strictly investigated which antimicrobial schemes are effective against KPC-producing Enterobacteriaceae isolates were few. Nevertheless, there were many retrospective studies in favor of the use of various combination therapy regimens against clinical $\mathrm{CPE}$ infections. None of these studies were investigated in Taiwan. The recommended antibiotic regimens included anti-pseudomonal carbapenem plus colistin or tigecycline, polymyxin plus tigecycline or 
aminoglycoside, or meropenem in combination with tigecycline and colistin. Important data regarding therapeutic efficacy of different antibiotic regimens (various combination schemes vs. monotherapy) for patients with CPE infections are illustrated in Table 2 (Lee and Burgess, 2012; Qureshi et al., 2012; Tumbarello et al., 2012; Tzouvelekis et al., 2012, 2014; Daikos et al., 2014; Food and Drug Administration [FDA], 2018; Rodríguez-Baño et al., 2018).

\section{OTHER ANTIMICROBIAL AGENTS AGAINST CPE INFECTIONS}

Apart from tigecycline and colistin against CPE, previous studies showed that avibactam, a bridged diazabicyclo octanone, exhibited good in vitro activity against GNB with serine $\beta$-lactamases (especially against the KPC producers) when combined with ceftazidime, but it is inactive against class $\mathrm{B}$ $\beta$-lactamases (Bonnefoy et al., 2004; Tzouvelekis et al., 2012; van Duin and Bonomo, 2016). In the Taiwan CDC-AMR 2017 survey, the MICs for ceftazidime-avibactam for one E. coli and one $K$. pneumoniae isolate that harbored the bla $a_{\mathrm{NDM}}$ allele were both $>64 \mathrm{mg} / \mathrm{L}$. Consequently, the Taiwanese 2017 data were consistent with previous studies. Ceftazidime-avibactam has been approved by the US-FDA in treatment of CRErelated cIAI and cUTI. In addition, meropenem-vaborbactam has an equivalent potency to ceftazidime-avibactam against class A carbapenemases (typified by KPC), but also has limited activity against class $\mathrm{B}$ along with oxacillinase carbapenemases (Castanheira et al., 2017; Chew et al., 2018). This drug was also approved in treatment of CRE-related cUTI recently. By contrast, aztreonam-avibactam displays good in vitro susceptibility in dual-carbapenemase (KPC, NDM)-producing CRE isolates (Chew et al., 2018). In addition, cefiderocol, a new siderophore cephalosporin, also shows potentially good in vitro activity against both KPC, as well as NDM-producing Enterobacteriaceae isolates (Hackel et al., 2018). The clinical trials comparing clinical efficacy of aztreonam-avibactam and cefiderocol with other best available antibiotics are undergone. The other future promising agents against CPE include imipenem-relebactam, plazomicin, and eravacycline (Rodríguez-Baño et al., 2018).

\section{ANTIBIOTIC TREATMENT FOR NON- CARBAPENEMASE-PRODUCING-CRE INFECTIONS}

Compared to ample databases regarding treatment of $\mathrm{CPE}$, limited data were available regarding treatment

\section{REFERENCES}

Bass, S. N., Bauer, S. R., Neuner, E. A., and Lam, S. W. (2015). Impact of combination antimicrobial therapy on mortality risk for critically ill patients with carbapenem-resistant bacteremia. Antimicrob. Agents Chemother. 59, 3748-3753. doi: 10.1128/AAC.00091-15 recommendations for clinical non-CP-CRE isolates. Nevertheless, Tamma et al. observed MIC values for imipenem and meropenem in the subgroup of CPE isolates ( $>90 \%$ were KPC producers) were significantly higher than those of the nonCP-CRE subgroup. Furthermore, the overall 14-day mortality rate was 4-fold higher among patients with CPE bacteremia than those of the non-CP-CRE group (Tamma et al., 2017). Recently, we investigated detection of CPE predictors among ertapenem-NS Enterobacteriaceae isolates causing IAI collected from patients hospitalized in Asia-Pacific countries during 2008-2014 (Jean et al.,2017). This Asia-Pacific IAI-CRE study showed that imipenem non-susceptibility (i.e., MIC $>2 \mathrm{mg} / \mathrm{L}$ ) and culturing ertapenem-NS Enterobacteriaceae isolates from the peritoneal space were highly likely as CPE (dominated by the $b l a_{\mathrm{NDM}}$ and $b l a_{\mathrm{IMP}}$-harboring isolates, rather than KPC producers; Jean et al., 2018a, in Infect Drug Resist). Moreover, the non-CP-ertapenem-NS IAI Enterobacteriaceae isolates were more likely to have cefepime MICs of $>8 \mathrm{mg} / \mathrm{L}$, which might be valuable in distinguishing it from the CPE. However, the clinical application of the dose adjustment of an anti-pseudomonal carbapenem agent to effectively treat the non-CP ertapenem-NS Enterobacteriaceae isolates needs further investigation in clinical scenarios.

\section{SUMMARY}

In Taiwan, the main research gap is that there are no sufficient clinical studies exploring the significant risk factors with respect to acquisition of CRE isolates among hospitalized or LTCF patients. Of note, the annual CPE proportion has been on a sharp rise among clinical CRE isolates since 2012, particularly for CR-K. pneumoniae isolates. To effectively limit the spread of CPE (especially ST11 K. pneumoniae clone) in clinical settings, close monitoring of this worrisome MDR trend is warranted at all major teaching hospitals, as well as nursing homes. In addition, although ceftazidime-avibactam and meropenemvaborbactam show excellent efficacy against some CPE, it is not currently available in Taiwan. Combination therapy schemes, such as colistin and/or tigecycline plus an anti-pseudomonal carbapenem agent, are still the preferred treatment for CRE and CPE infections in Taiwan.

\section{AUTHOR CONTRIBUTIONS}

S-SJ, N-YL, and P-RH collected and analyzed the data. S-SJ participated in the writing of the manuscript. S-SJ, N-YL, H-JT, $\mathrm{M}-\mathrm{CL}, \mathrm{W}-\mathrm{CK}$, and $\mathrm{P}-\mathrm{RH}$ read and approved the final version of the manuscript.

Bonnefoy, A., Dupuis-Hamelin, C., Steier, V., Delachaume, C., Seys, C., Stachyra, T., et al. (2004). In vitro activity of AVE1330A, an innovative broadspectrum non- $\beta$-lactam $\beta$-lactamase inhibitor. J. Antimicrob. Chemother. 54, 410-417. doi: 10.1093/jac/dkh358

Bratu, S., Landman, D., Haag, R., Recco, R., Eramo, A., Alam, M., et al. (2005a). Rapid spread of carbapenem-resistant Klebsiella pneumoniae in New York 
City: a new threat to our antibiotic armamentarium. Arch. Intern. Med. 165, 1430-1435. doi: 10.1001/archinte.165.12.1430

Bratu, S., Tolaney, P., Karumudi, U., Quale, J., Mooty, M., Nichani, S., et al. (2005b). Carbapenemase-producing Klebsiella pneumoniae in Brooklyn, NY: molecular epidemiology and in vitro activity of polymyxin B and other agents. J. Antimicrob. Chemother. 56, 128-132. doi: 10.1093/jac/dki175

Castanheira, M., Huband, M. D., Mendes, R. E., and Flamm, R. K. (2017). Meropenem-vaborbactam tested against contemporary Gram-negative isolates collected worldwide during 2014, including carbapenem-resistant, KPC-producing, multidrug-resistant, and extensively drug-resistant Enterobacteriaceae. Antimicrob. Agents Chemother. 61:e00567-17. doi: 10.1128/AAC.00567-17

Castanheira, M., Sader, H. S., Deshpande, L. M., Fritsche, T. R., and Jones, R. N. (2008). Antimicrobial activities of tigecycline and other broad-spectrum antimicrobials tested against serine carbapenemase- and metallo- $\beta$-lactamaseproducing Enterobacteriaceae: report from the SENTRY Antimicrobial Surveillance Program. Antimicrob. Agents Chemother. 52, 570-573. doi: 10. 1128/AAC.01114-07

Chang, Y. Y., Chuang, Y. C., Siu, L. K., Wu, T. L., Lin, J. C., Lu, P. L., et al. (2015). Clinical features of patients with carbapenem nonsusceptible Klebsiella pneumoniae and Escherichia coli in intensive care units: a nationwide multicenter study in Taiwan. J. Microbiol. Immunol. Infect. 48, 219-225. doi: 10.1016/j.jmii.2014.05.010

Chew, K. L., Tay, M. K. L., Cheng, B., Lin, R. T. P., Octavia, S., and Teo, J. W. P. (2018). Aztreonam-avibactam combination restores susceptibility of aztreonam in dual-carbapenemase-producing-Enterobacteriaceae. Antimicrob. Agents Chemother. 62, e414-e418. doi: 10.1128/AAC.00414-18

Chia, J. H., Siu, L. K., Su, L. H., Lin, H. S., Kuo, A. J., Lee, M. H., et al. (2009). Emergence of carbapenem-resistant Escherichia coli in Taiwan: resistance due to combined CMY-2 production and porin deficiency. J. Chemother. 21, 621-626. doi: 10.1179/joc.2009.21.6.621

Chiu, S. K., Huang, L. Y., Chen, H., Tsai, Y. K., Liou, C. H., Lin, J. C., et al. (2017). Roles of ramR and tet(A) mutations in conferring tigecycline resistance in carbapenem-resistant Klebsiella pneumoniae clinical isolates. Antimicrob. Agents Chemother. 61:e00391-17. doi: 10.1128/AAC.00391-17

Chiu, S. K., Ma, L., Chan, M. C., Lin, Y. T., Fung, C. P., Wu, T. L., et al. (2018). Carbapenem nonsusceptible Klebsiella pneumoniae in Taiwan: dissemination and increasing resistance of carbapenemase producers during 2012-2015. Sci. Rep. 8:8468. doi: 10.1038/s41598-018-26691-z

Chiu, S. K., Wu, T. L., Chuang, Y. C., Lin, J. C., Fung, C. P., Lu, P. L., et al. (2013). National surveillance study on carbapenem non-susceptible Klebsiella pneumoniae in Taiwan: the emergence and rapid dissemination of KPC-2 carbapenemase. PLoS One 8:e69428. doi: 10.1371/journal.pone.0069428

Chuang, C. Y. (2015). "An analysis of carbapenem resistant Enterobacteriaceae, associated nosocomial infections, and contact isolation measures," in Proceedings of the Abstracts of the 7th International Congress of the Asia Pacific Society of Infection Control, Taipei, 26-29. doi: 10.1016/j.jmii.2015.02.400

Clinical, and Laboratory Standards Institute [CLSI] (2018). Performance Standards for Antimicrobial Susceptibility Testing: Twenty-eighth Informational Supplement M100-S28. Wayne: CLSI.

Daikos, G. L., Tsaousi, S., Tzouvelekis, L. S., Anyfantis, I., Psichogiou, M., Argyropoulou, A., et al. (2014). Carbapenemase-producing Klebsiella pneumoniae bloodstream infections: lowering mortality by antibiotic combination schemes and the role of carbapenems. Antimicrob. Agents Chemother. 58, 2322-2328. doi: 10.1128/AAC.02166-13

Doumith, M., Ellington, M. J., Livermore, D. M., and Woodford, N. (2009). Molecular mechanisms disrupting porin expression in ertapenem-resistant Klebsiella and Enterobacter spp. clinical isolates from the UK. J. Antimicrob. Chemother. 63, 659-667. doi: 10.1093/jac/dkp029

Ejaz, H., Wang, N., Wilksch, J. J., Page, A. J., Cao, H., Gujaran, S., et al. (2017). Phylogenetic analysis of Klebsiella pneumoniae from hospitalized children. Pakistan. Emerg. Infect. Dis. 23, 1872-1875. doi: 10.3201/eid2311.1 70833

Endimiani, A., Patel, G., Hujer, K. M., Swaminathan, M., Perez, F., Rice, L. B., et al. (2010). In vitro activity of fosfomycin against blaKPC-containing Klebsiella pneumoniae isolates, including those nonsusceptible to tigecycline and/or colistin. Antimicrob. Agents Chemother. 54, 526-529. doi: 10.1128/AAC.0 1235-09
European Committee on Antimicrobial Susceptibility Testing [EUCAST] (2018). Breakpoint Tables for Interpretation of MICs and Zone Diameters, Version 5.0. Available at: http://www.eucast.org/clinical_points/ [accessed August 26, 2018] Food and Drug Administration [FDA] (2018). Prescribing Information For Tygacil (Tigecycline). Available at: http://www.accessdata.fda.gov/drugsatfda docs/label/2010/021821s021lbl.pdf [accessed August 16,2018]

Garonzik, S. M., Li, J., Thamlikitkul, V., Paterson, D. L., Shoham, S., Jacob, J., et al. (2011). Population pharmacokinetics of colistin methanesulfonate and formed colistin in critically ill patients from a multicenter study provide dosing suggestions for various categories of patients. Antimicrob. Agents Chemother. 55, 3284-3294. doi: 10.1128/AAC.01733-10

Girlich, D., Bouihat, N., Poirel, L., Benouda, A., and Nordmann, P. (2014). High rate of faecal carriage of extended-spectrum $\beta$-lactamase and OXA48 carbapenemase-producing Enterobacteriaceae at a university hospital in Morocco. Clin. Microbiol. Infect. 20, 350-354. doi: 10.1111/1469-0691. 12325

Gupta, N., Limbago, B. M., Patel, J. B., and Kallen, A. J. (2011). Carbapenemresistant Enterobacteriaceae: epidemiology and prevention. Clin. Infect. Dis. 53, 60-67. doi: 10.1093/cid/cir202

Hackel, M. A., Tsuji, M., Yamano, Y., Echols, R., Karlowsky, J. A., and Sahm, D. F. (2018). In vitro activity of the siderophore cephalosporin, cefiderocol, against carbapenem-nonsusceptible and multidrug-resistant isolates of Gram-negative bacilli collected worldwide in 2014 to 2016. Antimicrob. Agents Chemother. 62:e01968-17. doi: 10.1128/AAC.01968-17

Harris, P. N. A., Pezzani, M. D., Gutiérrez-Gutiérrez, B., Viale, P., Hsueh, P. R., Ruiz-Garbajosa, P., et al. (2017). Geographical variation in therapy for bloodstream infections due to multidrug-resistant Enterobacteriaceae: a posthoc analysis of the INCREMENT study. Int. J. Antimicrob. Agents 50, 664-672. doi: 10.1016/j.ijantimicag.2017.08.005

Ho, P. L., Cheung, Y. Y., Wang, Y., Lo, W. U., Lai, E. L., Chow, K. H., et al. (2016). Characterization of carbapenem-resistant Escherichia coli and Klebsiella pneumoniae from a healthcare region in Hong Kong. Eur. J. Clin. Microbiol. Infect. Dis. 35, 379-385. doi: 10.1007/s10096-015-2550-3

Huang, S. R., Liu, M. F., Lin, C. F., and Shi, Z. Y. (2014). Molecular surveillance and clinical outcomes of carbapenem-resistant Escherichia coli and Klebsiella pneumoniae infections. J. Microbiol. Immunol. Infect. 47, 187-196. doi: 10.1016/ j.jmii.2012.08.029

Jao, Y. T., Wang, W. H., Wang, A., Siu, L. K., and Lu, P. L. (2017). First report of OXA-48 carbapenemase-producing Escherichia coli in Taiwan. J. Microbiol. Immunol. Infect. 50, 403-404. doi: 10.1016/j.jmii.2016.12.003

Javed, H., Ejaz, H., Zafar, A., Rathore, A. W., and Haq, I. U. (2016). Metallo$\beta$-lactamase producing Escherichia coli and Klebsiella pneumoniae: a rising threat for hospitalized children. J. Pak. Med. Assoc. 66, 1068-1072.

Jean, S. S., Hsueh, P. R., and Smart Asia-Pacific Group. (2017). Distribution of ESBLs, AmpC $\beta$-lactamases and carbapenemases among Enterobacteriaceae isolates causing intra-abdominal and urinary tract infections in the Asia-Pacific region during 2008-14: results from the Study for Monitoring Antimicrobial Resistance Trends (SMART). J. Antimicrob. Chemother. 72, 166-171. doi: 10. 1093/jac/dkw398

Jean, S. S., Lee, W. S., and Hsueh, P. R. (2018a). Ertapenem non-susceptibility and independent predictors of the carbapenemase production among the Enterobacteriaceae isolates causing intra-abdominal infections in the AsiaPacific Region: results from the Study for Monitoring the Antimicrobial Resistance Trends (SMART). Infect. Drug Resist. 11, 1881-1891. doi: 10.2147/ IDR.S181085

Jean, S. S., Lee, W. S., Lam, C., Hsu, C. W., Chen, R. J., and Hsueh, P. R. (2015). Carbapenemase-producing Gram-negative bacteria: current epidemics, antimicrobial susceptibility and treatment options. Future Microbiol. 10, 407425. doi: $10.2217 / \mathrm{fmb} .14 .135$

Jean, S. S., Lu, M. C., Shi, Z. Y., Tseng, S. H., Wu, T. S., Lu, P. L., et al. (2018b). In vitro activity of ceftazidime-avibactam, ceftolozane-tazobactam, and other comparable agents against clinically important Gram-negative bacilli: results from the 2017 Surveillance of Multicenter Antimicrobial Resistance in Taiwan (SMART). Infect. Drug Resist. 11, 1983-1992. doi: 10.2147/IDR.S175679

Jernigan, M. G., Press, E. G., Nguyen, M. H., Clancy, C. J., and Shields, R. K. (2012). The combination of doripenem and colistin is bactericidal and synergistic against colistin-resistant, carbapenemase-producing Klebsiella pneumoniae. Antimicrob. Agents Chemother. 56, 3395-3398. doi: 10.1128/AAC.06364-11 
Khajuria, A., Praharaj, A. K., Kumar, M., and Grover, N. (2014). Carbapenem resistance among Enterobacter species in a tertiary care hospital in central India. Chemother. Res. Pract. 2014:972646. doi: 10.1155/2014/972646

Ku, Y. H., Chen, C. C., Lee, M. F., Chuang, Y. C., Tang, H. J., and Yu, W. L. (2017). Comparison of synergism between colistin, fosfomycin and tigecycline against extended-spectrum $\beta$-lactamase-producing Klebsiella pneumoniae isolates or with carbapenem resistance. J. Microbiol. Immunol. Infect. 50, 931-939. doi: 10.1016/j.jmii.2016.12.008

Kumarasamy, K. K., Toleman, M. A., Walsh, T. R., Bagaria, J., Butt, F., Balakrishnan, R., et al. (2010). Emergence of a new antibiotic resistance mechanism in India, Pakistan, and the UK: a molecular, biological, and epidemiological study. Lancet Infect. Dis. 10, 597-602. doi: 10.1016/S14733099(10)70143-2

Lai, C. C., Lin, T. L., Tseng, S. P., Huang, Y. T., Wang, J. T., Chang, S. C., et al. (2011). Pelvic abscess caused by New Delhi metallo- $\beta$-lactamase- 1 producing Klebsiella oxytoca in Taiwan in a patient who underwent renal transplantation in China. Diagn. Microbiol. Infect. Dis. 71, 474-475. doi: 10. 1016/j.diagmicrobio.2011.09.004

Lai, C. C., Lu, M. C., Tang, H. J., Chen, Y. H., Wu, Y. H., Chiang, H. T., et al. (2018). Implementation of a national quality improvement program to enhance hand hygiene in nursing homes in Taiwan. J. Microbiol. Immunol. Infect. doi: 10.1016/j.jmii.2018.09.007 [Epub ahead of print].

Lai, C. C., Wu, U. I., Wang, J. T., and Chang, S. C. (2013). Prevalence of carbapenemase-producing Enterobacteriaceae and its impact on clinical outcomes at a teaching hospital in Taiwan. J. Formos. Med. Assoc. 112, 492-496. doi: 10.1016/j.jfma.2012.09.021

Laolerd, W., Akeda, Y., Preeyanon, L., Ratthawongirakul, P., and Santanirand, P. (2018). Carbapenemase-producing carbapenem-resistant Enterobacteriaceae from Bangkok, Thailand, and their detection by the Carba NP and modified carbapenem inactivation method tests. Microb. Drug Resist. 24, 1006-1011. doi: $10.1089 / \mathrm{mdr} .2018 .0080$

Lee, C. M., Lai, C. C., Chiang, H. T., Lu, M. C., Wang, L. F., Tsai, T. L., et al. (2017). Presence of multidrug-resistant organisms in the residents and environments of long-term care facilities in Taiwan. J. Microbiol. Immunol. Infect. 50, 133-144. doi: 10.1016/j.jmii.2016.12.001

Lee, C. H., Su, T. Y., Ye, J. J., Hsu, P. C., Kuo, A. J., Chia, J. H., et al. (2017). Risk factors and clinical significance of bacteremia caused by Pseudomonas aeruginosa resistant only to carbapenems. J. Microbiol. Immunol. Infect. 50, 677-683. doi: 10.1016/j.jmii.2015.06.003

Lee, G. C., and Burgess, D. S. (2012). Treatment of Klebsiella pneumoniae carbapenemase (KPC) infections: a review of published case series and case reports. Ann. Clin. Microbiol. Antimicrob. 11:32. doi: 10.1186/1476-0711-11-32

Lee, J., Patel, G., Huprikar, S., Calfee, D. P., and Jenkins, S. G. (2009). Decreased susceptibility to polymyxin B during treatment for carbapenem-resistant Klebsiella pneumoniae infection. J. Clin. Microbiol. 47, 1611-1612. doi: 10.1128/ JCM.02466-08

Lee, M. Y., Ko, K. S., Kang, C. I., Chung, D. R., Peck, K. R., and Song, J. H. (2011). High prevalence of CTX-M-15-producing Klebsiella pneumoniae isolates in Asian countries: diverse clones and clonal dissemination. Int. J. Antimicrob. Agents 38, 160-163. doi: 10.1016/j.ijantimicag.2011.03.020

Liu, C. Y., Lai, C. C., Chiang, H. T., Lu, M. C., Wang, L. F., Tsai, T. L., et al. (2018). Predominance of methicillin-resistant Staphylococcus aureus in the residents and environments of long-term care facilities in Taiwan. J. Microbiol. Immunol. Infect. doi: 10.1016/j.jmii.2018.02.001 [Epub ahead of print].

Logan, L. K., and Weinstein, R. A. (2017). The epidemiology of carbapenemresistant Enterobacteriaceae: the impact and evolution of a global menace. J. Infect. Dis. 215, S28-S36. doi: 10.1093/infdis/jiw282

Lorenzoni, V. V., Silva, D. D. C., Rampelotto, R. F., Brites, P. C., Villa, B., and Hörner, R. (2017). Evaluation of carbapenem-resistant Enterobacteriaceae in a tertiary-level reference hospital in Rio Grande do Sul, Brazil. Rev. Soc. Bras. Med. Trop. 50, 685-688. doi: 10.1590/0037-8682-0209-2017

Ma, L., Lu, P. L., Siu, L. K., and Hsieh, M. H. (2013a). Molecular typing and resistance mechanisms of imipenem-non-susceptible Klebsiella pneumoniae in Taiwan: results from the Taiwan surveillance of antibiotic resistance (TSAR) study, 2002-2009. J. Med. Microbiol. 62, 101-107. doi: 10.1099/jmm.0.050492-0

Ma, L., Siu, L. K., Lin, J. C., Wu, T. L., Fung, C. P., Wang, J. T., et al. (2013b). Updated molecular epidemiology of carbapenem-non-susceptible Escherichia coli in Taiwan: first identification of KPC-2 or NDM-1-producing E. coli in Taiwan. BMC Infect. Dis. 13:599. doi: 10.1186/1471-2334-13-599

Mao, Y. C., Chang, C. L., Huang, Y. C., Su, L. H., and Lee, C. T. (2018). Laboratory investigation of a suspected outbreak caused by Providencia stuartii with intermediate resistance to imipenem at a long-term care facility. J. Microbiol. Immunol. Infect. 51, 214-219. doi: 10.1016/j.jmii.2016.07.004

Marchaim, D., Navon-Venezia, S., Schwaber, M. J., and Carmeli, Y. (2008). Isolation of imipenem-resistant Enterobacter species: emergence of KPC2 carbapenemase, molecular characterization, epidemiology, and outcomes. Antimicrob. Agents Chemother. 52, 1413-1418. doi: 10.1128/AAC.01103-07

Mariappan, S., Sekar, U., and Kamalanathan, A. (2017). Carbapenemase-producing Enterobacteriaceae: risk factors for infection and impact of resistance on outcomes. Int. J. Appl. Basic Med. Res. 7, 32-39. doi: 10.4103/2229-516X.198520

McConville, T. H., Sullivan, S. B., Gomez-Simmonds, A., Whittier, S., and Uhlemann, A. C. (2017). Carbapenem-resistant Enterobacteriaceae colonization (CRE) and subsequent risk of infection and 90-day mortality in critically ill patients, an observational study. PLoS One 12:e0186195. doi: 10. 1371/journal.pone.0186195

Mohanty, S., Gajanand, M., and Gaind, R. (2017). Identification of carbapenemasemediated resistance among Enterobacteriaceae bloodstream isolates: a molecular study from India. Indian J. Med. Microbiol. 35, 421-425. doi: 10.4103/ijmm.IJMM_16_386

Naas, T., Cuzon, G., Villegas, M. V., Lartigue, M. F., Quinn, J. P., and Nordmann, P. (2008). Genetic structures at the origin of acquisition of the $\beta$-lactamase blaKPC gene. Antimicrob. Agents Chemother. 52, 1257-1263. doi: 10.1128/AAC.0 1451-07

Navarro-San Francisco, C., Mora-Rillo, M., Romero-Gómez, M. P., MorenoRamos, F., Rico-Nieto, A., Ruiz-Carrascoso, G., et al. (2013). Bacteraemia due to OXA-48-carbapenemase-producing Enterobacteriaceae: a major clinical challenge. Clin. Microbiol. Infect. 19, E72-E79.

Nicolás, M. F., Ramos, P. I. P., Marques, de Carvalho, F., Camargo, D. R. A., de Fátima Morais, et al. (2018). Comparative genomic analysis of a clinical isolate of Klebsiella quasipneumoniae subsp. similipneumoniae, a KPC-2 and OKPB-6 beta-lactamases producer harboring two drug-resistance plasmids from southeast Brazil. Front. Microbiol. 9:220. doi: 10.3389/fmicb.2018.00220

Nordmann, P., Cuzon, G., and Naas, T. (2009). The real threat of Klebsiella pneumoniae carbapenemase-producing bacteria. Lancet Infect. Dis. 9, 228-236. doi: 10.1016/S1473-3099(09)70054-4

Quan, J., Li, X., Chen, Y., Jiang, Y., Zhou, Z., Zhang, H., et al. (2017). Prevalence of mcr-1 in Escherichia coli and Klebsiella pneumoniae recovered from bloodstream infections in China: a multicentre longitudinal study. Lancet Infect. Dis. 17, 400-410. doi: 10.1016/S1473-3099(16)30528-X

Qureshi, Z. A., Paterson, D. L., Potoski, B. A., Kilayko, M. C., Sandovsky, G., Sordillo, E., et al. (2012). Treatment outcome of bacteremia due to KPCproducing Klebsiella pneumoniae: superiority of combination antimicrobial regimens. Antimicrob. Agents Chemother. 56, 2108-2113. doi: 10.1128/AAC. 06268- 11

Rodríguez-Baño, J., Gutiérrez-Gutiérrez, B., Machuca, I., and Pascual, A. (2018). Treatment of infections caused by extended-spectrum- $\beta$-lactamase-, AmpC, and carbapenemase-producing Enterobacteriaceae. Clin. Microbiol. Rev. 31:e00079-17. doi: 10.1128/CMR.00079-17

Rui, Y., Lu, W., Li, S., Cheng, C., Sun, J., and Yang, Q. (2018). Integrons and insertion sequence common region 1 (ISCR1) of carbapenem-non-susceptible Gram-negative bacilli in fecal specimens from 5000 patients in southern China. Int. J. Antimicrob. Agents 52, 571-576. doi: 10.1016/j.ijantimicag.2018.06.015

Saïdani, M., Hammami, S., Kammoun, A., Slim, A., and Boutiba-Ben Boubaker, I. (2012). Emergence of carbapenem-resistant OXA-48 carbapenemaseproducing Enterobacteriaceae in Tunisia. J. Med. Microbiol. 61, 1746-1749. doi: 10.1099/jmm.0.045229-0

Sheu, C. C., Lin, S. Y., Chang, Y. T., Lee, C. Y., Chen, Y. H., and Hsueh, P. R. (2018). Management of infections caused by extended-spectrum $\beta$-lactamaseproducing Enterobacteriaceae: current evidence and future prospects. Expert Rev. Anti. Infect. Ther. 16, 205-218. doi: 10.1080/14787210.2018.1436966

Taiwan Nosocomial Infection Surveillance [TNIS] (2017). The Centers for Disease Control and Prevention, Taiwan. Available at: https://www.cdc.gov. tw/professional/downloadfile. aspx?fid=42C15EB49614A4B6 [accessed July 6, 2018] 
Tamma, P. D., Goodman, K. E., Harris, A. D., Tekle, T., Roberts, A., Taiwo, A., et al. (2017). Comparing the outcomes of patients with carbapenemase-producing and non-carbapenemase-producing carbapenem-resistant Enterobacteriaceae bacteremia. Clin. Infect. Dis. 64, 257-264. doi: 10.1093/cid/ciw741

Tang, H. J., Hsieh, C. F., Chang, P. C., Chen, J. J., Lin, Y. H., Lai, C. C., et al. (2016). Clinical significance of community- and healthcare-acquired carbapenem-resistant Enterobacteriaceae isolates. PLoS One 11:e0151897. doi: 10.1371/journal.pone.0151897

Thaden, J. T., Lewis, S. S., Hazen, K. C., Huslage, K., Fowler, V. G. Jr., Moehring, R. W., et al. (2014). Rising rates of carbapenem-resistant Enterobacteriaceae in community hospitals: a mixed-methods review of epidemiology and microbiology practices in a network of community hospitals in the southeastern United States. Infect. Control Hosp. Epidemiol. 35, 978-983. doi: 10.1086/677157

Tseng, S. P., Wang, S. F., Ma, L., Wang, T. Y., Yang, T. Y., Siu, L. K., et al. (2017). The plasmid-mediated fosfomycin resistance determinants and synergy of fosfomycin and meropenem in carbapenem-resistant Klebsiella pneumoniae isolates in Taiwan. J. Microbiol. Immunol. Infect. 50, 653-661. doi: 10.1016/j. jmii.2017.03.003

Tumbarello, M., Viale, P., Viscoli, C., Trecarichi, E. M., Tumietto, F., Marchese, A., et al. (2012). Predictors of mortality in bloodstream infections caused by Klebsiella pneumoniae carbapenemase-producing K. pneumoniae: importance of combination therapy. Clin. Infect. Dis. 55, 943-950. doi: 10.1093/cid/cis588

Tzouvelekis, L. S., Markogiannakis, A., Piperaki, E., Souli, M., and Daikos, G. L. (2014). Treating infections caused by carbapenemase-producing Enterobacteriaceae. Clin. Microbiol. Infect. 20, 862-872. doi: 10.1111/14690691.12697

Tzouvelekis, L. S., Markogiannakis, A., Psichogiou, M., Tassios, P. T., and Daikos, G. L. (2012). Carbapenemases in Klebsiella pneumoniae and other Enterobacteriaceae: an evolving crisis of global dimensions. Clin. Microbiol. Rev. 25, 682-707. doi: 10.1128/CMR.05035-11

van Duin, D., and Bonomo, R. A. (2016). Ceftazidime/avibactam and ceftolozane/tazobactam: second-generation $\beta$-Lactam $/ \beta$-Lactamase inhibitor combinations. Clin. Infect. Dis. 63, 234-241. doi: 10.1093/cid/ciw243

Wang, J. T., Wu, U. I., Lauderdale, T. L., Chen, M. C., Li, S. Y., Hsu, L. Y., et al. (2015). Carbapenem-nonsusceptible Enterobacteriaceae in Taiwan. PLoS One 10:e0121668. doi: 10.1371/journal.pone.0121668

Woodford, N., Xu-McCrae, L., Mushtaq, S., Wu, H. H. T., Ellington, M. J., Lancaster, O., et al. (2017). Prevalence of carbapenem resistance and carbapenemase production among Enterobacteriaceae isolated from urine in the UK: results of the UK infection-Carbapenem Resistance Evaluation Surveillance Trial (iCREST-UK). J. Antimicrob. Chemother. doi: 10.1093/jac/ $\mathrm{dkx} 471$ [Epub ahead of print].

World Health Organization [WHO] (2017). Global Priority List of AntibioticResistant Bacteria to Guide Research, Discovery and Development of New Antibiotics. Available at: http://www.who.int/medicines/publications/globalpriority-list-antibiotic-resistant-bacteria/en/ [accessed August 16,2018]

Wu, H. S., Chen, T. L., Chen, I. C., Huang, M. S., Wang, F. D., Fung, C. P., et al. (2010). First identification of a patient colonized with Klebsiella pneumoniae carrying blaNDM-1 in Taiwan. J. Chin. Med. Assoc. 73, 596-598. doi: 10.1016/ S1726-4901(10)70129-5

Wu, P. F., Chuang, C., Su, C. F., Lin, Y. T., Chan, Y. J., Wang, F. D., et al. (2016). High minimum inhibitory concentration of imipenem as a predictor of fatal outcome in patients with carbapenem non-susceptible Klebsiella pneumoniae. Sci. Rep. 6, 32665. doi: 10.1038/srep32665

Yahav, D., Lador, A., Paul, M., and Leibovici, L. (2011). Efficacy and safety of tigecycline: a systematic review and meta-analysis. J. Antimicrob. Chemother. 66, 1963-1971. doi: 10.1093/jac/dkr242

Yang, F. C., Yan, J. J., Hung, K. H., and Wu, J. J. (2012). Characterization of ertapenem-resistant Enterobacter cloacae in a Taiwanese university hospital. J. Clin. Microbiol. 50, 223-226. doi: 10.1128/JCM.01263-11

Yang, T. Y., Lu, P. L., and Tseng, S. P. (2017). Update on fosfomycin-modified genes in Enterobacteriaceae. J. Microbiol. Immunol. Infect. doi: 10.1016/j.jmii.2017.10. 006 [Epub ahead of print].

Zhang, R., Liu, L., Zhou, H., Chan, E. W., Li, J., Fang, Y., et al. (2017). Nationwide surveillance of clinical carbapenem-resistant Enterobacteriaceae (CRE) strains in China. EBioMedicine 19, 98-106. doi: 10.1016/j.ebiom.2017.04.032

Conflict of Interest Statement: The authors declare that the research was conducted in the absence of any commercial or financial relationships that could be construed as a potential conflict of interest.

Copyright (c) 2018 Jean, Lee, Tang, Lu, Ko and Hsueh. This is an open-access article distributed under the terms of the Creative Commons Attribution License (CC BY). The use, distribution or reproduction in other forums is permitted, provided the original author(s) and the copyright owner(s) are credited and that the original publication in this journal is cited, in accordance with accepted academic practice. No use, distribution or reproduction is permitted which does not comply with these terms. 